\title{
VIOLÊNCIA INTRAFAMILIAR E COMUNITÁRIA CONTRA MULHERES: UM PROBLEMA DE SAÚDE PÚBLICA
}

\author{
INTRAFAMILIAR AND COMMUNITARY VIOLENCE AGAINST WOMEN: \\ A PUBLIC HEALTH PROBLEM
}

\author{
Tayná Ribeiro Monteiro de Figueiredo ${ }^{1}$ \\ Ítalo de Macedo Bernardino ${ }^{2}$ \\ Lorena Marques da Nóbrega ${ }^{3}$ \\ Sérgio D’Ávila Lins Bezerra Cavalcanti ${ }^{4}$
}

RESUMO: INTRODUÇÃO: O reconhecimento da violência contra a mulher como um importante problema de saúde pública e uma grave violação dos direitos humanos faz-se necessário para que haja alocação adequada de recursos para a expansão dos centros de atenção social voltados às vítimas de violência. OBJETIVO: Objetivou-se com esse estudo traçar o perfil da violência intrafamiliar e comunitária contra mulher de acordo com as características sociodemográficas das vítimas e dos agressores excluindo os casos de parceiros íntimos. MÉTODOS: Foi realizado um estudo observacional, de caráter exploratório, com dados secundários oriundos do Núcleo de Medicina e Odontologia Legal (NUMOL) de um município do Nordeste brasileiro. Realizou-se a análise estatística descritiva objetivando caracterizar a amostra. Em seguida, empregou-se a análise de diferença de proporções (teste quiquadrado de Pearson ou teste exato de Fisher quando apropriado) para determinar associação entre mecanismo de agressão e demais variáveis investigadas. RESULTADOS: A maioria da amostra tinha entre 30 e 59 anos $(32,0 \%)$, era solteira $(63,6 \%)$, possuía baixa escolaridade $(65,6 \%)$ e não trabalhava $(45,9 \%)$. O agressor em sua maioria era do sexo masculino $(52,7 \%)$, sendo geralmente familiar da vítima $(96,6 \%)$. Prevaleceram situações de agressões sem instrumento (80,4\%), sendo registradas principalmente aos domingos $(18,3 \%)$ e à noite $(38,7 \%)$. CONCLUSÃO: Portanto, observou-se associação estatística significativa entre mecanismo de agressão e escolaridade, ocupação da vítima, sexo do agressor e período de ocorrência. Dessa forma, foi possível observar um perfil da violência intrafamiliar e

\footnotetext{
${ }^{1}$ Mestranda pelo departamento de Odontologia da Universidade Estadual da Paraíba - Campina Grande (PB), Brasil. Autor correspondente: Tayná Ribeiro Monteiro de Figueiredo. Departamento de Odontologia da Universidade Estadual da Paraíba. Rua Baraúnas, 351, Bairro Universitário, CEP: 58429-500, Campina Grande, PB, Brasil. E- mail: taynaribeirof@hotmail.com.

2 Doutorando pelo departamento de Odontologia da Universidade Estadual da Paraíba.

3 Professora substituta pelo departamento de Odontologia da Universidade Estadual da Paraíba

${ }^{4}$ Professor efetivo pelo departamento de Odontologia da Universidade Estadual da Paraíba.
} 
comunitária contra a mulher de acordo com as características sociodemográficas das vítimas e dos agressores.

Palavras chave: Violência; Violência contra a Mulher; Saúde Pública, Epidemiologia.

ABSTRACT: INTRODUCTION: Recognition of violence against women as a major public health and a serious violation of human rights is necessary for adequate allocation of resources for the expansion of social care centers for victims of violence. OBJECTIVE: The goal of this study was to trace the intrafamiliar and communitary violence profile against women, based on victim's sociodemographic characteristics and their agressors, excluding close partners cases. METHODS: An observacional study of exploratory character was realized, the data was obtained from Núcleo de Medicina e Odontologia Legal (NUMOL) of one municipality in Northeast of Brazil. Descriptive statistical analysis were carried in order to classify the samples. Then, a proportion difference analysis (Pearson's chi-squared test or Fisher's exact test when appropriate) was performed to identify association between aggression mechanisms and other variables that were investigated. RESULTS: Most of the samples were 30 to 59 years old (32,0\%), single women $(63,6 \%)$, low school level $(65,6 \%)$, and were unemployed (45,9\%). The aggressor was, mostly, a man $(52,7 \%)$, generally close to the victim (96,6\%). Results shown that aggressive cases without instrument prevailed (80,4\%), usually occurring on Sundays (18,3\%), in night hours (38,7\%). CONCLUSION: We conclude a significant statistical association between aggressive mechanisms and school level, victim occupation, sex of the aggressor, aggressive mechanisms and occurrence period. Thus, it was possible to identify an intrafamiliar and communitary violence profile against women based on victim's sociodemographic characteristics and their agressors.

Keywords: Violence; Violence against Women; Public health; Epidemiology. 


\section{INTRODUÇÃO}

A violência intrafamiliar e comunitária é um tema que tem atraído à atenção de governantes, estudiosos, bem como da sociedade geral (SILVA, OLIVEIRA, 2015). Não obstante, o número de casos notificados tem crescido cada vez mais (QUADROS et al., 2013). A Organização Mundial da Saúde (OMS) estima que, em todo o mundo, uma em cada três mulheres é ou já foi vítima de violência doméstica (DV), gerando grandes desafios para os sistemas de saúde (KRUG, MISERICÓRDIA, DAHLBERG, 2002).

Observa-se que as consequências da violência são todas danosas, como um todo, à saúde da mulher vítima de violência intrafamiliar ou comunitária. Segundo Ribeiro e Coutinho (2011), a vivência da violência diminui drasticamente a qualidade de vida dessas mulheres, atingindo negativamente sua saúde física, psicológica e principalmente a social, fazendo as vítimas se isolarem e perderem gradativamente sua rede de apoio, tornando-se vulneráveis e com poucas estratégias de enfrentamento, sendo cada vez mais difícil quebrar este ciclo. Dessa forma, a violência contra a mulher é caracterizada por sua invisibilidade, visto que a maioria dessas ocorrências não termina em assistência e não é registrada nos sistemas de informação, resultando em uma subnotificação dos eventos (NEVES, GARCIA, 2016; GARCIA et al., 2016; OMS, 2013; GARCIA, 2016).

Dados nacionais mostram que pessoas que sofreram violência doméstica e familiar apresentam maior risco de problemas de saúde física e psicológica do que a população em geral, afetando significativamente os gastos com saúde. Além disso, também está associada ao absenteísmo no trabalho e na escola (OMS, 2002; BRASIL, 2009). No Mapa da Violência, o Brasil ficou em quinto lugar entre os países com maior taxa de homicídio por 100.000 mulheres em 2013 (VIEIRA, PERDONA, SANTOS, 2011).

O aumento das vítimas femininas mostra-se contrário à tentativa do governo federal de impedir o crescimento dos abusos contra as mulheres, considerando que 
a Lei $n^{0} 11.340$ (2006) foi criada com o objetivo de diminuir a violência doméstica. Apesar disso, o Relatório do Estado da Paraíba sobre a violência, argumenta que esse aumento pode ser aparente, e pode estar relacionado a uma maior disseminação da importância na denúncia de casos de violência e ao aumento do empoderamento das mulheres para buscar apoio nas redes assistenciais (BARBOSA et al., 2019).

O reconhecimento da violência contra a mulher como um importante problema de saúde pública e uma grave violação dos direitos humanos faz-se necessário para que haja alocação adequada de recursos para a expansão dos centros de atenção social voltados às vítimas de violência. O encaminhamento adequado e oportuno da mulher agredida, e seu acesso aos serviços de segurança pública e justiça para a obtenção de medidas protetivas podem fazer a diferença entre a vida e a morte (GARCIA, 2016).

Apesar dos problemas associados à violência doméstica e familiar, o conhecimento sobre sua distribuição está ausente. Poucos estudos investigaram os fatores associados a esse tipo de violência no país (VIEIRA, PERDONA, SANTOS, 2011; AUDI et al., 2008; D'OLIVEIRA et al., 2009). Desta forma, mais estudos nacionais com tal perspectiva podem contribuir com a comunidade científica e acadêmica no sentido de produzir melhores e maiores conhecimentos da subjetivação do fenômeno da violência doméstica e comunitária contra a mulher. Sendo assim, podem colaborar para novas possibilidades de intervenções, assim como implementação de políticas públicas destinadas à vigilância, prevenção e assistência social (FONSECA, RIBEIRO, LEAL, 2012).

Este estudo objetivou traçar o perfil da violência intrafamiliar e comunitária contra mulher de acordo com as características sociodemográficas das vítimas e dos agressores. 


\section{METODOLOGIA}

Foi realizado um estudo observacional, de caráter exploratório, com dados secundários oriundos do Núcleo de Medicina e Odontologia Legal (NUMOL) de uma região metropolitana do Nordeste do Brasil, que possui uma população estimada em aproximadamente 687.545 habitantes (IBGE, 2010), sendo considerada a segunda maior zona metropolitana do interior nordestino, quarta maior zona metropolitana do interior brasileiro, abrangendo 15 municípios, dentre eles o município de Campina Grande e demais municípios adjacentes.

Foram adotados como critérios de inclusão todos os registros das vítimas que sofreram agressão física e foram submetidas a exame de corpo de delito, registrado ao longo de um período de quatro anos (Janeiro de 2008 a Dezembro de 2011). Como critérios de exclusão foram excluídos os registros que estavam indisponíveis no momento da coleta por questões judiciais. Além disso, como o preenchimento dos registros é manuscrito, os manuscritos que estavam ilegíveis, mesmo após uma consulta a um funcionário do setor, também foram excluídos. Foram excluídos também os dados cujo agressor era o parceiro íntimo da vitima ou ex-parceiro.

Para a coleta dos dados, elaborou-se um formulário específico que continham variáveis relacionadas às características sociodemográficas das vítimas (idade, escolaridade, ocupação, estado civil), dos agressores (sexo, ligação com a vítima) e às características da violência (mecanismo de agressão, região do corpo afetada, dia e turno de ocorrência).

Previamente à coleta, foi feito um estudo piloto objetivando adequar o formulário a ser utilizado. Três componentes da equipe foram submetidos aos procedimentos de calibração para a coleta de dados. As concordâncias intraexaminador e inter-examinador foram avaliadas mediante o teste Kappa e ambas obtiveram $\mathrm{K}=0,85-0,90$, consideradas muito boas, habilitando os examinadores a realizarem o estudo.

Inicialmente, realizou-se a análise estatística descritiva objetivando caracterizar a amostra. Em seguida, empregou-se a análise de diferença de 
proporções (teste qui-quadrado de Pearson ou teste exato de Fisher quando apropriado) para determinar associação entre mecanismo de agressão e demais variáveis investigadas. O nível de significância foi fixado em $p<0,05$ (HAIR et al., 2009). Todas as análises foram conduzidas com auxílio do software IBM SPSS Statistics versão 20.0, considerando um intervalo de confiança de 95\%.

O desenvolvimento desse estudo seguiu os princípios éticos da pesquisa envolvendo seres humados, conforme pressupõe a Resolução 196/96. O projeto foi registrado no SISNEP (Sistema Nacional de Informações sobre Ética em Pesquisa envolvendo Seres Humados) avaliado por um Comitê de Ética em Pesquisa de forma independente, sendo aprovado (CAAE: 02266.0.133.000-10). Todos os

direitos das vítimas foram protegidos e seguidos os preceitos nacionais $\mathrm{e}$ internacionais de ética em pesquisa com seres humanos.

\section{RESULTADOS}

Durante o período investigado, foram registrados 409 casos de violência doméstica contra a mulher efetuada por uma pessoa do âmbito familiar ou conhecido, excluindo o companheiro ou ex-companheiro.

A distribuição das vítimas de acordo com as características sociodemográficas e características dos agressores. A maioria das vítimas tinha entre 30 e 59 anos (32,0\%), era solteira (63,6\%), possuía baixa escolaridade $(65,6 \%)$ e não trabalhava (45,9\%). O agressor em sua maioria era do sexo masculino $(52,7 \%)$, sendo geralmente familiar da vítima $(96,6 \%)$ (Tabela 1$)$. 
Tabela 1. Distribuição das mulheres vítimas de violência doméstica de acordo com as características sociodemográficas e características dos agressores (2008 a 2011).

\begin{tabular}{lcc}
\hline Variáveis & $\mathbf{n}$ & \% \\
\hline Faixa etária da vítima & 35 & 8,7 \\
0 a 9 & 104 & 25,8 \\
10 a 19 & 117 & 29,0 \\
20 a 29 & 129 & 32,0 \\
30 a 59 & 18 & 4,5 \\
60 ou mais & & \\
Estado civil da vítima & 253 & 63,6 \\
Solteiro(a) & 12 & 3,0 \\
Viúvo(a) & 21 & 5,3 \\
Separado(a) & 76 & 19,1 \\
Casado(a) & 36 & 9,0 \\
União Estável & & \\
Escolaridade da vítima & 208 & 65,6 \\
S8 anos de estudo & 109 & 34,4 \\
> 8 anos de estudo & & \\
Ocupação da vítima & 158 & 45,9 \\
Não trabalha & 117 & 34,0 \\
Assalariada & 69 & 20,1 \\
Não assalariada & & \\
Sexo do agressor & 187 & 47,3 \\
Feminino & 208 & 52,7 \\
Masculino & & \\
Relação entre agressor e vítima & 395 & 96,6 \\
Familiar & 14 & 3,4 \\
Conhecido & &
\end{tabular}

Nota. Diferenças na categoria total devido a missing data.

A distribuição das mulheres vítimas de violência doméstica de acordo com o mecanismo de agressão, período de ocorrência e região do corpo afetada. Prevaleceram situações de agressões sem instrumento, usando força física $(80,4 \%)$, sendo registradas principalmente aos domingos $(18,3 \%)$ e no turno da noite $(38,7 \%)$. Traumas em mais de uma região do corpo constituíram o padrão mais frequente $(50,6 \%)$, seguido de casos de traumas isolados na região da cabeça $(19,1 \%)$ (Tabela 2). 
Tabela 2. Distribuição das mulheres vítimas de violência doméstica de acordo com o mecanismo de agressão, período de ocorrência e região do corpo afetada (2008 a 2011).

\begin{tabular}{|c|c|c|}
\hline Variáveis & $\mathbf{n}$ & $\%$ \\
\hline \multicolumn{3}{|l|}{ Mecanismo de agressão } \\
\hline Agressões sem instrumento & 311 & 80,4 \\
\hline Arma de fogo & 2 & 0,5 \\
\hline Arma branca & 20 & 5,2 \\
\hline Outros & 40 & 10,3 \\
\hline Mista & 14 & 3,6 \\
\hline \multicolumn{3}{|l|}{ Dia da ocorrência } \\
\hline Segunda & 62 & 16,0 \\
\hline Terça & 48 & 12,4 \\
\hline Quarta & 56 & 14,4 \\
\hline Quinta & 53 & 13,7 \\
\hline Sexta & 58 & 14,9 \\
\hline Sábado & 40 & 10,3 \\
\hline Domingo & 71 & 18,3 \\
\hline \multicolumn{3}{|l|}{ Horário da ocorrência } \\
\hline 00:00-05:59 & 13 & 3,6 \\
\hline $06: 00-11: 59$ & 78 & 21,5 \\
\hline $12: 00-17: 59$ & 131 & 36,2 \\
\hline $18: 00-23: 59$ & 140 & 38,7 \\
\hline \multicolumn{3}{|l|}{ Região do corpo afetada } \\
\hline Cabeça & 78 & 19,1 \\
\hline Pescoço & 9 & 2,2 \\
\hline Membro Superior & 77 & 18,8 \\
\hline Membro Inferior & 19 & 4,6 \\
\hline Tórax & 13 & 3,2 \\
\hline Abdome & 6 & 1,5 \\
\hline Mais de uma região & 207 & 50,6 \\
\hline
\end{tabular}

Nota. Diferenças na categoria total devido a missing data.

Quanto à análise bivariada entre o mecanismo de agressão e demais variáveis investigadas. Verificou-se associação estatisticamente significativa entre agressões com uso de instrumentos, grupo etário mais jovem $(p=0,021)$, solteiras $(p=0,036)$, que não trabalhavam $(p=0,020)$ e ocorrências no turno da madrugada $(p=0,021)$. 
Tabela 3. Análise bivariada entre o mecanismo de agressão e demais variáveis investigadas.

\begin{tabular}{|c|c|c|c|c|c|c|c|}
\hline \multicolumn{8}{|c|}{ Mecanismo de agressão } \\
\hline \multirow[t]{2}{*}{ Variáveis } & \multicolumn{2}{|c|}{$\begin{array}{c}\text { Uso de } \\
\text { instrumentos }\end{array}$} & \multicolumn{2}{|c|}{$\begin{array}{l}\text { Agressões } \\
\text { nuas }\end{array}$} & \multicolumn{2}{|c|}{ Total } & \multirow[t]{2}{*}{ p-valor } \\
\hline & $\mathbf{n}$ & $\%$ & $\mathbf{n}$ & $\%$ & $\mathbf{n}$ & $\%$ & \\
\hline Faixa etária & & & & & & & $0,021^{(1) *}$ \\
\hline 0 a 9 & 12 & 34,3 & 23 & 65,7 & 35 & 100,0 & \\
\hline 10 a 19 & 24 & 24,5 & 74 & 75,5 & 98 & 100,0 & \\
\hline 20 a 29 & 16 & 14,4 & 95 & 85,6 & 111 & 100,0 & \\
\hline 30 a 59 & 17 & 14,2 & 103 & 85,8 & 120 & 100,0 & \\
\hline 60 ou mais & 5 & 29,4 & 12 & 70,6 & 17 & 100,0 & \\
\hline Estado civil & & & & & & & $0,036^{(2) *}$ \\
\hline Solteiro(a) & 58 & 24,1 & 183 & 75,9 & 241 & 100,0 & \\
\hline Viúvo(a) & 1 & 11,1 & 8 & 88,9 & 9 & 100,0 & \\
\hline Separado(a) & 3 & 14,3 & 18 & 85,7 & 21 & 100,0 & \\
\hline Casado(a) & 6 & 8,3 & 66 & 91,7 & 72 & 100,0 & \\
\hline União Estável & 6 & 17,6 & 28 & 82,4 & 34 & 100,0 & \\
\hline Escolaridade da vítima & & & & & & & $0,138^{(1)}$ \\
\hline Não alfabetizado & 6 & 37,5 & 10 & 62,5 & 16 & 100,0 & \\
\hline Ensino Fundamental & 41 & 22,3 & 143 & 77,7 & 184 & 100,0 & \\
\hline Ensino Médio & 10 & 14,1 & 61 & 85,9 & 71 & 100,0 & \\
\hline Ensino Superior & 6 & 18,2 & 27 & 81,8 & 33 & 100,0 & \\
\hline Ocupação & & & & & & & $0,020^{(1) *}$ \\
\hline Não trabalha & 40 & 26,7 & 110 & 73,3 & 150 & 100,0 & \\
\hline Assalariado & 15 & 13,4 & 97 & 86,6 & 112 & 100,0 & \\
\hline Não assaliariado & 10 & 16,1 & 52 & 83,9 & 62 & 100,0 & \\
\hline Sexo do Agressor & & & & & & & $0,556^{(1)}$ \\
\hline Feminino & 32 & 18,3 & 143 & 81,7 & 175 & 100,0 & \\
\hline Masculino & 41 & 20,7 & 157 & 79,3 & 198 & 100,0 & \\
\hline Sujeito agressor & & & & & & & $0,474^{(2)}$ \\
\hline Familiar & 75 & 20,0 & 300 & 80,0 & 375 & 100,0 & \\
\hline Conhecido & 1 & 8,3 & 11 & 91,7 & 12 & 100,0 & \\
\hline Dia da ocorrência & & & & & & & $0,547^{(2)}$ \\
\hline Segunda & 12 & 20,3 & 47 & 79,7 & 59 & 100,0 & \\
\hline Terça & 7 & 15,2 & 39 & 84,8 & 46 & 100,0 & \\
\hline Quarta & 11 & 21,2 & 41 & 78,8 & 52 & 100,0 & \\
\hline Quinta & 10 & 20,4 & 39 & 79,6 & 49 & 100,0 & \\
\hline Sexta & 11 & 19,6 & 45 & 80,4 & 56 & 100,0 & \\
\hline Sábado & 3 & 8,1 & 34 & 91,9 & 37 & 100,0 & \\
\hline Domingo & 17 & 24,3 & 53 & 75,7 & 70 & 100,0 & \\
\hline Horário da ocorrência & & & & & & & $0,021^{(2)}$ \\
\hline $00: 00-05: 59$ & 4 & 30,8 & 9 & 69,2 & 13 & 100,0 & \\
\hline $06: 00-11: 59$ & 15 & 20,0 & 60 & 80,0 & 75 & 100,0 & \\
\hline $12: 00-17: 59$ & 12 & 9,8 & 110 & 90,2 & 122 & 100,0 & \\
\hline
\end{tabular}


18:00-23:59

Região do corpo

Cabeça e face

Pescoço

Membro superior

Membro inferior

Tórax

Abdome

Mais de uma região
$30 \quad 22,2 \quad 105 \quad 77,8 \quad 135 \quad 100,0$

$\begin{array}{llll}\mathbf{0 , 7 7 5 ^ { ( 2 ) }} & \end{array}$

$\begin{array}{llllll}11 & 14,7 & 64 & 85,3 & 75 & 100,0\end{array}$

$\begin{array}{llllll}2 & 25,0 & 6 & 75,0 & 8 & 100,0\end{array}$

$\begin{array}{llllll}17 & 23,6 & 55 & 76,4 & 72 & 100,0\end{array}$

$\begin{array}{llllll}4 & 25,0 & 12 & 75,0 & 16 & 100,0\end{array}$

$\begin{array}{llllll}3 & 23,1 & 10 & 76,9 & 13 & 100,0\end{array}$

$\begin{array}{llllll}1 & 20,0 & 4 & 80,0 & 5 & 100,0\end{array}$

$\begin{array}{llllll}38 & 19,2 & 160 & 80,8 & 198 & 100,0\end{array}$

Nota $^{(1)}$ Teste qui-quadrado de Pearson; ${ }^{(2)}$ Teste exato de Fisher; ${ }^{*} p<0,05$.

\section{DISCUSSÃO}

Neste trabalho, a faixa etária de 30 a 59 anos foi a mais acometida. Em concordância, estudo feito no Paquistão por Ali et al. (2011) mostrou que mulheres mais velhas apresentam um risco de sofrer violência física maior do que as mais jovens, onde foi observado a média de idade das vítimas de 35,72, aproximando-se da média de idade encontrada em estudo desenvolvido na Nigéria por Ajah et al. (2014), que foi de 30,9 anos. Entretanto, a literatura revela evidências que observaram a faixa etária de 18 a 29 anos como a mais frequente (GARCIA et al., 2016; LEITE et al., 2017).

Uma hipótese levantada para explicar esses achados é que, à medida que as mulheres envelhecem, elas se tornam economicamente mais produtivas e socialmente influentes, tendo mais oportunidade de se desvencilhar da condição abusiva, tornando-se então menos propensas a registrar violência recente do que as mulheres mais jovens (NAVED, PERSSO, 2005; KISHOR, JOHNSON, 2004). Entretanto, baseado na mesma hipótese que mulheres mais velhas tornam-se mais produtivas economicamente e menos dependentes, estas são mais propensas a denunciar, visto que não possuem dependência econômica e tendem a ter menos medo quando comparado a mulheres mais jovens, o que facilita ao desprendimento desta condição e menor índice de subnotificação, devido ao menor receio de denunciar. 
Foi relatado maior vitimização por agressão física em mulheres com escolaridade $\leq 8$ anos de estudo e que não trabalham. Os achados do presente trabalho foram consistentes com aqueles de outros estudos realizados no Brasil (GARCIA et al., 2016; LEITE et al., 2017; MATTOS, RIBEIRO, CAMARGO, 2012). De acordo com a literatura, há uma relação direta entre baixa escolaridade e maior propensão em as mulheres reportarem violência (D'OLIVEIRA et al., 2009; ALI, KRANTZ, MOGREN, 2012; RADA, 2014; BECK et al., 2014); indicando a condição de menor escolaridade como fator de risco para a violência (GARCIA et al., 2016; NAVED, PERSSO, 2005). Tal relação pode ser explicada pela condição de maior grau de educação estar intimamente ligado ao maior acesso à informação e aos recursos, o que conduz a menor tolerância a um relacionamento de caráter abusivo (BECK et al., 2014).

Sobressaíram os agressores do sexo masculino, consistente a achados prévios da literatura em que as mulheres são mais agredidas por homens, os quais na maioria das vezes possuem alguma relação íntima com a vítima (AJAH et al., 2014; SILVA, OLIVEIRA, 2016). Quanto à relação entre agressor e vítima, quase todos os agressores eram familiares da vítima. Este estudo foi o primeiro estudo da literatura com enfoque na violência contra as mulheres perpetrada por familiar ou conhecido, excluindo o parceiro e ex-parceiro íntimo da vítima. Foi observado na literatura um percentual estatisticamente relevante de agressores cuja relação com a vítima é de caráter familiar ou conhecido da vítima, porém há poucos estudos que se aprofundaram a respeito, visto que o foco maior tem sido na violência doméstica acometida por parceiro íntimo. A partir desse estudo, observamos a necessidade de um olhar mais atento para as vítimas além do contexto conjugal, visto que a violência contra a mulher é um problema multicausal e multifacetado.

Com relação ao mecanismo de agressão e região do corpo afetada. Prevaleceram situações de agressões sem instrumento, usando força física, semelhante ao estudo de Garcia et al. (2016), Lamoglia e Minayo (2009) e Silva e Oliveira (2016). Traumas em mais de uma região do corpo constituíram o padrão mais frequente, seguido de casos de traumas isolados na região da cabeça. Já em estudo realizado por Garcia et al. (2016), as partes do corpo mais frequentemente atingidas foram a cabeça, seguida por membros superiores e múltiplos órgãos; 
enquanto Silva e Oliveira (2016) observaram os órgãos genitais como parte do corpo mais afetada, seguido por cabeça/face.

O fato de a cabeça ser relatada como parte do corpo mais atingida pode ser explicada devido a sua localização proeminente e sua exposição e falta de proteção. Além disso, o valor estético do rosto para as mulheres faz com que seja o principal alvo do ataque na tentativa de intimidar e humilhar as vítimas. Os membros superiores também estão entre as áreas mais afetadas do corpo, visto que poderiam ser usados para autodefesa, proteção e minimização das lesões resultantes dos conflitos (NÓBREGA et al., 2016).

Ao associar o mecanismo de agressão e demais variáveis, foi constatada associação estatisticamente significativa entre agressões com uso de instrumentos, grupo etário mais jovem, solteiras, que não trabalhavam e ocorrências no turno da madrugada. Tais achados tiveram concordância com um estudo realizado no Rio de Janeiro, onde houve associação significativa de vítimas mais jovens, escolaridade baixa, não exercício de atividade remunerada e ocorrências nos finais de semana em turno noturno ou madrugada (GARCIA et al., 2016). De forma semelhante, Nóbrega et al. (2016) observou em seu estudo a prevalência de vítimas jovens, solteiras, com pouca educação e dependentes financeiramente de outras pessoas. Baseado nisso, pode-se constatar que mulheres economicamente e socialmente desfavorecidas tornam-se um grupo vulnerável a comportamentos agressivos.

Dadas as consequências e prevalência desse trauma, os estudos devem examinar o perfil das populações de risco e suas experiências no combate à violência contra as mulheres, uma vez que tal violência é evitável. Este panorama atual de caráter nacional e internacional demanda um olhar urgente de todos os níveis de organização política, social, judicial e da saúde (BERNARDINO, 2016; NÓBREGA et al., 2016).

O presente estudo realizou uma abordagem pontual do problema da violência contra a mulher, mas foi possível observar a necessidade de discussão de novas questões que poderão ser abordadas em pesquisas futuras. O alto índice de subnotificação foi uma limitação encontrada no estudo, visto que a violência contra a mulher é caracterizada por sua invisibilidade, já que muitas dessas ocorrências não terminam em assistência e não é registrada nos sistemas de informação. Contudo, o 
objetivo proposto foi alcançado, uma vez que foi possível observar um perfil da violência intrafamiliar e comunitária contra a mulher de acordo com as características sociodemográficas das vítimas e dos agressores.

\section{CONCLUSÃO}

Verificou-se que existe associação estatística significativa entre mecanismo de agressão e escolaridade e ocupação da vítima, sexo do agressor e período de ocorrência. Dessa forma, foi possível observar um perfil da violência intrafamiliar e comunitária contra a mulher de acordo com as características sociodemográficas das vítimas e dos agressores, onde predominaram vítimas jovens, solteiras, que não trabalhavam e violência caracterizada por uso de instrumentos e ocorrência no turno da madrugada.

\section{REFERÊNCIAS BIBLIOGRÁFICAS}

AJAH, L.O.; IYOKE, C.A.; NKWO, P.O.; NWAKOBY, B.; EZEONU, P. Comparison of domestic violence against women in urban versus rural areas of southeast Nigeria. Internacional Journal Womens Health. v. 6, p. 865-872, 2014.

ALI, T.S.; ASAD, N.; MOGREN, I.; KRANTZ, G. Intimate partner violence in urban Pakistan: prevalence, frequency, and risk factors. Internacional Journal Womens Health. v. 3, p. 105115, 2011.

ALI, T.S.; KRANTZ, G.; MOGREN, I. Violence permeating daily life: a qualitative study investigating perspectives on violence among women in Karachi, Pakistan. Internacional Journal Womens Health. v. 4, p. 577-585, 2012.

AUDI, C.A.F.; SEGALL-CORREAA, A.M.; SANTIAGO, S.M.; ANDRADE, M.G.G.; PÉREZESCAMILA, R. Violence against pregnant women: prevalence and associated factors. Revista Saúde Pública. v. 42, p. 877-885, 2008.

BARBOSA, K.; RODRIGUES, L.; ALENCAR, G.; D'AVILA, S.; FERREIRA, E.; FERREIRA, R. Explorando o efeito do tempo e do sexo na violência familiar e comunitária de 2008 a 2014. Revista De Saúde Pública, v. 53, n. 46, 2019.

BECK, J.G.; CLAPP, J.D.; JACOBS-LENTZ, J.; MCNIFF, J.; AVERY, M., OLSEN, S.A. The association of mental health conditions with employment, interpersonal, and subjective functioning after intimate partner violence. Violence Against Women. v. 20, n. 11, p. 13211337, 2014. 
BERNARDINO, I.M.; BARBOSA, K.G.N.; NOBREGA, L.M.; CAVALCANTE, G.M. S.; FERREIRA, E.F.E.; D'AVILA, S. Violence against women in different stages of the life cycle in Brazil: an exploratory study. Revista Brasileira de Epidemiologia (Online), v. 19, p. 740-752, 2016.

Brazilian Institute of Geography and Statistics: First Results of the 2010 Census. [http://cidades.ibge.gov.br/xtras/home.php].

D'OLIVEIRA, A.F.P.L.; SCHRAIBER, L.B.; FRANÇA-JUNIOR, I.; LUDERMIR, A.B.; PORTELLA, A.P.; DINIZ, C.S. et al. Factors associated with intimate partner violence against Brazilian women. Revista Saúde Pública. v. 43, p. 299-311, 2009.

FONSECA, D. H.; RIBEIRO, C. G.; LEAL, N. S. B. Violência doméstica contra a mulher: realidades e representações sociais. Psicologia \& Sociedade. v. 24, n. 2, p. 307-314, 2012.

GARCIA, L.P.; DUARTE, C.E.; FREITAS, L.R.S.; SILVA, G.D.M. Violência doméstica e familiar contra a mulher: estudo de casos e controles com vítimas atendidas em serviços de urgência e emergência. Cad Saude Publica. v. 32, n. 4, 2016.

GARCIA, Leila Posenato. The invisible magnitude of violence against women. Epidemiologia Serviço Saúde, Brasília, v. 25, n. 3, p. 451-454, 2016.

HAIR, J.F.; BLACK, W.C.; BABIN, J.B.; ANDERSON, R.E.; TATHAM, R.L. Multivariate Data Analysis. 7th edition. Prentice-Hall: Copyright; 2009.

KISHOR, S.; JOHNSON, K. Profiling domestic violence: a multi-country study. Stud Fam Plann. v. 36, p. $259-261,2004$.

KRUG, E.G.; MISERICÓRDIA, J.A.; DAHLBERG LL, Z.W.I. AB. O relatório mundial sobre violência e saúde. Lancet. v. 360, n. 9339, p. 1083-1088, 2002.

LAMOGLIA, C.V.A.; MINAYO, M.C.S. Violência conjugal, um problema social e de saúde pública: estudo em uma delegacia do interior do Estado do Rio de Janeiro. Ciencia Saude Coletiva. v. 14, n. 2, p. 595-604, 2009.

LEITE, F.M.C.; AMORIM, M.H.C.; WEHRMEISTER, F. C.; GIGANTE, D.P. Violência contra mulheres, Espírito Santo, Brasil. Revista Saude Publica. v. 51, p. 33, 2017.

MATTOS, P.R.; RIBEIRO, I.S.; CAMARGO, V.C. Análise dos casos notificados de violência contra mulher. Cogitare Enferm. v. 17, n. 4, p. 738-44, 2012.

MINISTÉRIO DA SAÚDE DO BRASIL. Instrução para preenchimento da ficha de notificação / investigação de violência doméstica, sexual e / ou outros. Brasília: Ministério da Saúde do Brasil; 2009.

NAVED, R.T.; PERSSO, L.A. Factors associated with spousal physical violence against women in Bangladesh. Stud Fam Plann. v. 36, p. 289-300, 2005.

NEVES, A.C.M.; GARCIA, L.P. Atendimentos de jovens de agressões em serviços públicos de urgência e emergência, 2011: diferenças entre sexos. Ciencia Saude Coletiva. 2016.

NOBREGA, L.M.; BERNARDINO, I.M.; GRANVILLE-GARCIA, A.F.; CAVALCANTE, G.M.S.; FERREIRA, E.F.E.; D'AVILA, S. Characteristics of violence against women in Campina Grande, north-eastern Brazil. Tanzania journal of health research, v. 18, p. 55-65, 2016.

ORGANIZAÇÃO MUNDIAL DA SAÚDE (OMS). Relatório mundial sobre violência e saúde. Brasília (DF): OMS / OPAS; 2002. 
ORGANIZAÇÃO MUNDIAL DA SAÚDE. Estimativas globais e regionais da violência contra as mulheres: prevalência e efeitos na saúde da violência praticada pelo parceiro íntimo e da violência sexual não relacionada. Genebra: Organização Mundial da Saúde; 2013.

QUADROS, M.E.F.; OLIVEIRA, M.M.; FERREIRA, R.Z.; ALVES, P.F. Violência doméstica: caracterização e atitude da equipe de saúde da família frente à problemática. Revista Enfermagem UFSM. v. 3, n. 1, p. 164-174, 2013.

RADA, C. Violence against women by male partners and against children within the family: prevalence, associated factors, and intergenerational transmission in Romania, a crosssectional study. BMC Public Health. v. 14, p. 129, 2014.

RIBEIRO, C. G.; COUTINHO, M. L. L. Representações sociais de mulheres vítimas de violência doméstica na cidade de João Pessoa-PB. Psicologia e Saúde, v. 3, n. 1, p. 52-59. 2011.

SILVA, L.E.L.; OLIVEIRA, M.L.C. Epidemiological characteristics of violence against women in the Federal District, Brazil, 2009-2012. Epidemiologia Serviço Saúde, Brasília, v. 25, n. 2, p. 331-342, 2016.

SILVA, L.E.L.; OLIVEIRA, M.L.C. Violence against women: systematic review of the Brazilian scientific literature within the period from 2009 to 2013. Ciencia Saude Coletiva.v. 20, n. 11, p. 3523-3532, 2015.

VIEIRA, E.M.; PERDONA, G.S.C.; SANTOS, M.A. Factors associated with intimate partner physical violence among health service users. Revista Saúde Pública. v. 45, p. 730-737, 2011. 